(171)

\title{
Bacterial Remediation of Ciprofloxacin (CIP) by CIP Resistance Bacteria Isolated from Environment Samples
}

\author{
Liyanage G.Y., Manage P.M.* \\ Centre for Water Quality and Algae Research, Department of Zoology, \\ University of Sri Jayewardenepura, Sri Lanka \\ *pathmalal@sjp.ac.lk
}

\begin{abstract}
Ciprofloxacin (CIP) is a fluoroquinolone antibacterial drug widely used in treatment of pathogenic infectious diseases in human. A significant quantity of CIP is metabolised incompletely by patients after administration and enter to the environment with patients' excretion. Therefore, studies on the biodegradability of drugs can be taken as a very first step of an environmental risk assessment. The present study reports the biodegradation of CIP by Lactobacillus gesseri, Enterobacter sp., Bacillus sp., Bacillus subtilius and Micrococcus luteus which were isolated as CIP resistance, non-pathogenic bacteria. The presence of antibiotic-resistant bacteria was identified using the 16s rRNA sequencing. A $0.5 \mathrm{ml}$ of overnight starved bacterial suspension was introduced into medium containing CIP at final concentration of $5 \mathrm{ppm}$. Triplicate samples were incubated at $280^{\circ} \mathrm{C}$ with shaking at $100 \mathrm{ppm}$. A $0.5 \mathrm{ml}$ of subsample was removed at 2 days interval for a period of 14 days. Samples were subjected to High Performance Liquid Chromatography (HPLC) analysis. Fourier Transform Infrared Spectroscopy (FTIR) analyses were carried out for each sample at the end of the 14 days to find structures of by-products. Complete degradation of CIP by L. gesseri was detected at the end of 14 days of incubation with average degradation rate of $0.182 \pm 0.15 \mathrm{~d}^{-1}$. Descending degradation rates were followed by Enterobacter sp. $\left(0.75 \pm 0.03 \mathrm{~d}^{-1}\right)$ and Bacillus sp. $\left(0.41 \pm 0.02 \mathrm{~d}^{-1}\right)$ at 8 and 6 days respectively. However, clear cut degradation of CIP was not detected for B. subtilis and $M$. luteus respectively. Further, FTIR spectrum revealed that incubation of L. gesseri, Enterobacter sp. and Bacillus sp., breakdown the piperazine ring and quinolone part in the CIP structure while degradation occurred.
\end{abstract}

Keywords: Ciprofloxacin; Degradation, Fourier Transform Infrared Spectroscopy (FTIR), High Performance Liquid Chromatography (HPLC); Lactobacillus gesseri 